Nevada. It may be of interest to compare the chlorine found in Owens Lake with that present in various other saline waters. The average chlorine for the ocean is about igoo parts per I,000,000; Big Soda Lake has 40,000 parts; Great Salt Lake (in 1873) 73,000; Urmiah Lake, I20,000; Bogdo Lake, 160,000; Indevsk Lake, I60,000; Elton Lake, I70,000; the Dead Sea, in I864, a maximum of 170,000 ; and two salt springs in Oklahoma, recently examined by one of us, over 180,000 parts. Thus it will be seen that all the above-named waters, with the exception of the ocean and Big Soda Lake, have a higher chlorine content than Owens Lake, and exactly the same relation holds with regard to the total solids.

While no general conclusions can be drawn from the results of a single analysis, this one has at least shown that the water of Owens Lake is rapidly concentrating and at the present rate will soon reach the saturation point. It has also demonstrated the existence in determinable quantities of certain substances which have hitherto been neglected. We believe that if a similar analysis could be made, at regular intervals, of several of the inportant saline bodies of water, such as Great Salt Lake, Owens Lake, Soda Lake, etc., interesting and valuable data would be accumulated, and it is our hope to be able to undertake a small portion of such work in the near future.

U. S. Geological Strkver:

BERKELEY, CAL.

[CoNtribution from the Whlicome Chemical Research LaboraTORIES, LONDON.]

\title{
CHEMICAL AND PHYSIOLOGICAL EXAMINATION OF THE FRUIT OF CHAILLETIA TOXICARIA. ${ }^{1}$
}

(A WEST AFRICAN POISON.)

BY FREDERICK B. POWER AND FRANK TUTIN.

Received June I9, 1906.

THE present investigation of the fruit of Chailletia toxicaria, Don, was undertaken at the suggestion of Dr. W. Renner, Medical Officer, Colonial Hospital, Freetown, Sierra Leone, who also kindly provided us with the material for this purpose.

The interest which this subject possesses will be apparent from

1 Presented, in abstract, at the International Congress of Applied Chemistry, Rome, Italy, April, Igo6. 
the following abstract of a paper published by Dr. Renner, entitled "Native Poison, West Africa."1

"For some years now, death by poison has been the subject of talk in the Colony of Sierra Leone, and one could scarcely credit the statements so often made with respect to this subject. No one, it would appear, dies from natural causes. Poisoning in one form or another is put down as the cause of death, not only among the poor but also among the rich, and yet no one would or could come forward to attest the fact that such a one had been poisoned by such a substance. Some of these deaths which appear to be mysterious have given cause for great alarm and anxiety, and aroused a feeling of dread and bitterness against the country doctors to whom they are attributed.

"A peculiar kind of disease, of the origin of which no account could be given, is now and again met with among the people. The European doctor, when called in, is puzzled, for he sees before him a young healthy man or woman, between the ages of twentythree and forty years, struck down with paralysis. He examines the case carefully, cross-examines scrupulously the patient and his friends, and fails to arrive at any conclusion from these as to the source of the trouble. He suspects poison, and is told that the person has been poisoned. He endeavors to treat the symptoms, and either fails or the case is removed to a country doctor. The individual may recover or die, this depending on the nature and the quantity of the poison administered. These cases are brought forward daily. It has been my fortune lately to discover the cause of one of the mysterious and sudden diseases that affects the natives in the form of paralysis of the lower limbs."

A description is given by Dr. Renner of a case of poisoning, which occurred with a native laborer through his having eaten some fish on which the ground fruit of Chailletia toxicaria had been strewn for the purpose of killing rats, and the article then continues as follows:

"The Chailletia toxicaria belongs to the natural order of Chail letiacee, and is known as 'Ratsbane.' In the Colony proper it is called 'Broken Back.' By the Mendis of the Hinterland it is known under the name of 'Magbevi,' and by the Timnes as

1 J. African Society, 1904, pp. I09-III. Compare also The British Medical Journal, June 4, I904, p. I3I4. 
'Manăk.' It grows plentifully in West Africa and South America, and the fruit, which contains the poisonous substance, is used largely for the destruction of rats and other animals; but beyond this it is also used extensively by the people in the Colony and the Hinterland to poison one another. Conversing with some of the older men of the Timnes and Mendis, I found that with them it is very frequently used against their enemies to poison wellwater or streams which supply hostile villages. Domestic animals poisoned by it are seen to rush about in great excitement as if in severe pain; they vomit, and drag their hind limbs, which ultimately become paralyzed. The forearm and chest muscles become paralyzed also, and they die from paralysis of the respiratory centre."

In the "Flora of Tropical Africa," by Daniel Oliver, F.R.S., London, I868, Vol. I, p. 34I, a complete description is given of the plant in question, from which the following, including references to its synonymy and habitat, is abstracted:

"Chailletia toxicaria, Don, DC. Prod. II, 57. A glabrous shrub or the young shoots and inflorescence mealy-puberulous at first. Leaves rather coriaceous .... 2 $2 \frac{1}{2}-6$ inches long, $1 \frac{1}{4}-2 \frac{1}{4}$ inches broad, occasionally rather larger. Fruit $\mathrm{I}-\mathrm{I} \frac{1}{2}$ inches long, ellipsoidal, hard and woody, one-celled, one-seeded.

"Synonym.-C. affnis, Planch. F1. Nigrit 276.

"Habitat.--Upper Guinea, Sierra Leone, and Senegambia."

The "Treasury of Botany," by Lindley and Moore, London, I 899, Vol. I, p. 260, also gives a description of Chailletia, together with the following information:

"A genus which is found more or less in most tropical countries, but represented in greatest numbers in Brazil. The only extratropical species is $C$. cymosa, which is a native of South Africa; and the only species whose uses are recorded is $C$. toxicaria, a native of Sierra Leone, where the seeds of this plant are said to be used by the colonists for poisoning rats and by them called ratsbane. Upwards of thirty species are known."

The above-mentioned Chailletia cymosa is of special interest in this connection, as will be evident from the following notice of it recorded in the "Bulletin of the Imperial Institute," 1903, Vo1. I, p. 14. "Preliminary experiments have shown that the leaves furnish prussic acid when powdered and moistened with 
water, and from them a cyanogenetic glucoside has been isolated, and is being further examined."

A description of this species of Chailletia is given in the "Flora Capensis," by Harvey and Sonder, Dublin, I859-I860, Vol. I, p. 450 , from which the following is abstracted:

"Chailletia cymosa (Hook. Ic. Pl.t. 591). Habitat, Aapges River, Burke and Zeyher! Oct. (Herb. Hook., T. C. D.). A very dwarf shrub or suffrutex, under a foot in height. Leaves $3-3 \frac{1}{2}$ inches long, 6-1o lines wide, etc. Fruit unknown. A remarkable plant, very different in habit from others of the genus, but apparently not differing generically, unless the fruit afford a character."

\section{EXPERIMENTAL.}

The material received by us from Dr. Renner, of Sierra Leone, corresponded with the description of the fruit of Chailletia toxicaria, Don, as recorded in Oliver's "Flora of Tropical Africa,"1 with the exception that it is occasionally two-celled and twoseeded. This is also the case with authentic specimens of these fruits in the Museum of Economic Botany at Kew. The weight of the individual dry fruits ranges from about 5 to 8 grams.

The following preliminary experiments were conducted.

Test for Alkaloid.-Ten grams of the powdered fruit were digested with Prollius' fluid, and the resulting liquid filtered and evaporated. The residue, extracted with acidulated water, afforded no reaction with the usual alkaloid reagents.

Test for a Cyanogenetic Glucoside.-As it has been observed by other investigators ${ }^{1}$ that the leaves of Chailletia cymosa contain a cyanogenetic glucoside, the fruit of $C$. toxicaria was specially tested for a substance of this class. Ten grams of the ground fruit were mixed with a little water and allowed to stand in a tightly corked flask for about twenty-four hours. As no odor of hydrocyanic acid was then apparent, an emulsion of sweet almonds was added, but this produced no evident change. The liquid was finally distilled, and the complete absence of hydrocyanic acid in the distillate was established by chemical tests. It could, therefore, be concluded that the fruit of Chailletia toxicaria does not contain a cyanogenetic compound, and this conclusion was confirmed by the more complete examination of

I Lac, cit. 
a very much larger quantity of the fruit, which will subsequently be described.

Test for a Poisonous Proteid.-It was considered possible that the poisonous action of the fruit might be due to a proteid substance, such, for example, as ricin, abrin or robin. Fifty grams of the ground fruit were therefore digested with water at the ordinary temperature for three days, when the mixture was brought upon a strainer, expressed, and the liquid filtered. The clear filtrate afforded, however, no reaction for a soluble proteid.

Extraction with Solvents.-In order to obtain some further preliminary information respecting the character of the drug, Ioo grams of it, in fine powder, were extracted successively in a Soxhlet apparatus with various solvents. The amounts of extract obtained, after drying in a steam-oven until of constant weight, were as follows:

\begin{tabular}{|c|c|c|}
\hline \multicolumn{2}{|c|}{ Petroleum (b. p. $40-60^{\circ}$ ) extracted } & $\begin{array}{l}\text { Per cent. } \\
2.00\end{array}$ \\
\hline Ether & “ & 0.26 \\
\hline Chloroform & “ & I. 10 \\
\hline Ethyl acetate & “" & 3.04 \\
\hline Alcohol & “ & 6.84 \\
\hline
\end{tabular}

For the purpose of a systematic examination of the constituents of the Chailletia fruit, 3000 grams of it, ground to a fine powder, were first extracted in a percolator with cold light petroleum, and subsequently with hot alcohol.

\section{A. EXAMINATION OF THE PETROLEUM EXTRACT.}

This was a yellowish brown fat, which, at ordinary temperatures, consisted of a mixture of an oily substance and a granular solid, but when exposed to cold became almost completely solid. The total amount of fatty matter was 55 grams, corresponding to I.83 per cent. of the weight of the fruit. The solid was separated from the oil by filtration at the pump, then dissolved in hot acetone, some alcohol added, and the solution allowed to stand for a time. A little fluffy matter first separated, which was removed, and the fat was then deposited in the form of white nodules. These were separated by filtration, washed with alcohol, dried on a tile, then dissolved in a large quantity of absolute alcohol, in which they were sparingly soluble, and the solution filtered. When the liquid was nearly cold, the fat separated in white, crystalline 
nodules, which were removed by filtration. After drying on a tile, a portion was finally dried in a vacuum desiccator, when it melted at $42-43^{\circ}$. Another portion was dried by heating in a steam-oven, when, after solidification, it melted at $35-40^{\circ}$. The whole of the substance was then recrystallized from absolute alcohol, when it melted sharply at $43^{\circ}$. Its solution in chloroform readily absorbed bromine.

0.1450 gram gave 0.4072 gram $\mathrm{CO}_{2}$ and $0.157 \mathrm{I}$ gram $\mathrm{H}_{2} \mathrm{O}$. $\mathrm{C}=76.6 ; \mathrm{H}=\mathrm{I} 2.0 ; \mathrm{C}_{3} \mathrm{H}_{5} .\left(\mathrm{C}_{18} \mathrm{H}_{35} \mathrm{O}_{2}\right)_{2} \cdot \mathrm{C}_{18} \mathrm{H}_{33} \mathrm{O}_{2}$ requires $\mathrm{C}=77.0 ;$ $\mathrm{H}=\mathrm{I} 2 . \mathrm{I}$ per cent. 0.2499 gram absorbed 0.0686 gram I. Hence the iodine value $=27.5$.

This crystalline substance was evidently oleodistearin, which has a calculated iodine value of 28.6 , and is stated to melt at $44^{\circ}{ }^{1}$ Further proof of its identity was afforded by the following experiment: I.6 grams of the substance were hydrolyzed with an alcoholic solution of potassium hydroxide, and the acids thus obtained were fractionally crystallized many times. The most readily soluble fraction consisted of an oil which was found to be unsaturated, on treatment with nitrous acid formed a solid mass, and therefore consisted of oleic acid. The other fractions melted at $69-70^{\circ}$, and consisted entirely of stearic acid, as shown by the following analysis:

0.1049 gram gave 0.2912 gram $\mathrm{CO}_{2}$ and 0.1198 gram $\mathrm{H}_{2} \mathrm{O}$. $\mathrm{C}=75.7 ; \mathrm{H}=12.7 ; \mathrm{C}_{18} \mathrm{H}_{38} \mathrm{O}_{2}$ requires $\mathrm{C}=76.0 ; \mathrm{H}=12.7$ per cent.

The identification of the mixed triglyceride, oleodistearin, as a constituent of the fatty oil from Chailletia fruit is of considerable interest, inasmuch as the number of substances of this class that have been isolated from either vegetable or animal fats and definitely characterized is comparatively small (compare Lewkowitsch's "Chemical Technology and Analysis of Oils, Fats, and Waxes," third edition, Vol. I, p. I4).

The original filtrate from the granular solid which afforded the oleodistearin consisted of a brownish oil, which became partially solid on cold days. This, together with the fatty matter contained in the first and second mother-liquors from the recrystallization of the oleodistearin, was hydrolyzed by boiling with an alcoholic solution of potassium hydroxide. The alcohol was then removed, the alkaline residue mixed with clean sand, dried, and extracted in a Soxhlet apparatus with light petroleum. On

${ }^{1}$ Ber. 32, 388 (1899). Compare also Chem. Centr. I, 1716 (1905). 
removing the petroleum from this extract a dark brown resinous residue was obtained, which was dissolved in much alcohol, and the liquid boiled with animal charcoal and filtered. On cooling, a small quantity (about 0.5 gram) of a substance was deposited in the form of crystalline plates. This was removed by filtration, recrystallized several times from ethyl acetate, and then from ordinary spirit, in which it was very sparingly soluble. It was then almost colorless, and, after drying at $100^{\circ}$, melted at $138-143^{\circ}$. After again crystallizing from spirit, and drying at $100^{\circ}$, when it appeared to lose some water of crystallization, it softened and gradually melted at $135^{-1} 48^{\circ}$.

0.0854 gram, dried at $100^{\circ}$, gave 0.2630 gram $\mathrm{CO}_{2}$ and $0.095^{2}$ gram $\mathrm{H}_{2} \mathrm{O} . \quad \mathrm{C}=84.0 ; \mathrm{H}=\mathrm{I} 2.4 ; \mathrm{C}_{2 \mathrm{H}} \mathrm{H}_{44} \mathrm{O}$ requires $\mathrm{C}=83.9 ; \mathrm{H}=\mathrm{I}$ I .8 per cent.

This substance was evidently a phytosterol, since it also afforded the color reactions characteristic of the latter when dissolved in chloroform and treated with acetic anhydride and sulphuric acid. As the melting-point was not sharp, it was possibly a mixture of substances of that class, but the amount was too small to admit of a further separation.

The alkaline mixture with sand, from which the phytosterol had been extracted, was treated with hot water in order to dissolve the potassium salts, the solution then acidified with sulphuric acid, and distilled in steam. A clear distillate was obtained, containing only a small amount of acids, which, after converting into their barium salts, were found to consist chiefly of formic acid with a little butyric acid. The non-volatile acids remaining in the flask were removed by shaking the liquid repeatedly with ether. The ethereal liquid was washed with water, dried with calcium chloride, the ether then removed, and the residue dissolved in hot spirit. After as much as possible of the solid acids had been removed by crystallization, the oily acids which separated from the mother-liquors were redissolved in alcohol and completely precipitated as lead salts by the addition of a solution of lead acetate in dilute alcohol. These lead salts were collected on a filter, washed with alcohol, dried, and then extracted with ether, when the greater portion dissolved. From the portion of lead salts which remained undissolved by this treatment the acids were regenerated, and, being solid, they were added to the above-mentioned solid acids which had previously 
been separated by crystallization. The whole was then fractionally crystallized, when the most sparingly soluble fractions were found to consist of stearic acid (melting-point $69^{\circ}$ ), while the more readily soluble ones appeared to be a mixture of stearic and palmitic acids, and melted at $53-60^{\circ}$.

From the above-mentioned portion of the lead salts which had been dissolved by ether the acids were likewise regenerated. An oily liquid was thus obtained, a portion of which, when treated with nitrous acid, became converted into a solid mass, thus indicating the presence of oleic acid. The remainder of this liquid was distilled under diminished pressure, when a pale yellow oil was obtained, from which no solid matter separated.

0.2508 gram absorbed 0.2696 gram I. Hence the iodine value $=107.5$.

As oleic acid has a calculated iodine value of 90.I, it was evidently associated with some acid possessing a higher degree of unsaturation.

\section{B. EXAMINATION OF THE ALCOHOL EXTRACT,}

It has already been stated that the Chailletia fruit, after having been extracted with light petroleum, was extracted with hot alcohol. This alcoholic extract was concentrated and mixed with water, in order to precipitate the resin. The mixture was warmed to expel the remaining alcohol, allowed to cool, and the insoluble resinous substance removed by filtration, washed with hot water and dried.

I. The Resin.-This formed a dry, brown, amorphous powder, having an odor somewhat resembling that of chocolate. The amount of resinous substance was 76 grams, corresponding to 2.5 per cent. of the weight of the fruit. It appeared to be slightly soluble in water, as a further quantity was deposited on concentrating the aqueous liquid. After keeping for some time it lost its original smell, and became much more sparingly soluble in alcohol. In order to ascertain whether any definite substance could be obtained from it, it was mixed with prepared sawdust, and extracted successively in a Soxhlet apparatus with chloroform, ethyl acetate, and alcohol. The amounts of extract obtained by these solvents, after drying in a steam oven, were as follows : 
(a) Chloroform Extract, consisting of a dark green, soft resin, I I. 83 grams.

(b) Ethyl Acetate Extract, a yellowish brown, very sticky resin, 4.30 grams.

(c) Alcohol Extract, a nearly hard, brown resin, 9.40 grams.

These results showed the resin to consist of a complex mixture, and, as after the final extraction with alcohol about two-thirds of the original substance remained undissolved in the form of a brown powder, it was evident that it had undergone some change, either in drying or in the process of extraction. As there appeared to be nothing crystalline in these products, they were not examined chemically, but were reserved for physiological tests.

II. The Aqueous Liquid.-The liquid from which the abovedescribed resinous substances had been separated had a brownish color, gave a black coloration with ferric chloride, a dense, grayish colored precipitate with basic lead acetate, and reduced Fehling's solution. In order to purify it, basic lead acetate was added until no further precipitate was produced. This precipitate was collected by filtration at the pump, washed with a little water, and then suspended in water and decomposed by hydrogen sulphide. The filtrate from the precipitated lead sulphide formed a clear, dark brown liquid, which was concentrated under diminished pressure. A thick, dark brown syrup was thus obtained, which consisted chiefly of tannic and coloring-matters, and deposited nothing crystalline, even after long standing.

The aqueous filtrate from the basic lead acetate precipitate was completely deprived of lead by means of hydrogen sulphide, and then concentrated under diminished pressure to the consistency of a thick syrup. When heated with potassium hydroxide it evolved ammonia, and it readily reduced Fehling's solution. An aqueous solution of the syrup was slightly levorotatory, and afforded an osazone melting at $214^{\circ}$. The sugar, therefore, appeared to be chiefly $i$-glucose, the osazone of which is stated to melt at $217-218^{\circ}$.

PHYSIOLOGICAL TESTS AND ATTEMPTS TO ISOLATE THE POISONOUS PRINCIPLE.

The indefinite character of the products obtained from the original alcoholic extract $B$, as above described, rendered it important at this stage of the investigation that we should ascertain 
which of them represented or contained the poisonous principle. For this purpose some physiological experiments were conducted for us, with essentially the following results.

I. The Resin. - The three extracts obtained from this by successive treatment with different solvents, as previously described, were separately tested. In each case 0.5 gram of the extract was administered to a small dog.

(a) Chloroform Extract.-This, after some hours, produced narcosis, and ultimately death, but did not cause the delirium and epileptiform convulsions which are such characteristic features of the poisonous action of Chailletia fruit.

(b) Ethyl Acetate Extract.-This produced the characteristic convulsions, thus differing in its action from the preceding extract.

(c) Alcohol Extract.-This caused nausea, but no toxic symptoms.

II. The Aqueous Liquid.-This liquid, from which the abovementioned resinous substances had been separated, and which had been further purified by means of basic lead acetate, was found to be highly toxic. When concentrated to a syrupy consistency it amounted to about 200 grams. Of this syrup 0.5 gram was administered to a dog, when after an interval of three hours the characteristic convulsions occurred, which were followed by death within fifteen minutes. The decomposed lead acetate compound was also toxic, and when given in the same amount as the syrup produced a similar effect, although somewhat more slowly. This result, however, was probably due to the occlusion of some of the aqueous liquid with the lead acetate precipitate, as the latter did not permit of being very thoroughly washed.

The preceding physiological experiments having shown that the syrupy aqueous liquid possessed in a high degree the poisonous properties of the Chailletia fruit, and as nothing crystalline separated from it, even after standing for several weeks, an attempt was made to isolate the toxic principle by further treatment. The syrup was accordingly mixed with prepared sawdust, the mixture thoroughly dried in a vacuum over sulphuric acid, then brought into a Soxhlet apparatus, and extracted for two days with dry ethyl acetate. This removed a quantity of substance which was only sparingly soluble in the ethyl acetate, for it separated from the hot liquid as a brownish syrup. The liquid 
decanted from the latter was concentrated to a small volume, when another portion of syrup separated, which was removed. From the remaining liquid the ethyl acetate was for the most part distilled off, when from the resulting syrup an exceedingly small quantity of a colorless, crystalline substance separated in the form of needles. The syrup containing these needles was diluted with a little alcohol and filtered, and a very small amount of the crystalline substance was thus obtained. This was only sparingly soluble in ethyl acetate and in alcohol, and practically insoluble in water. After recrystallization from hot alcohol, and drying at $100^{\circ}$, it melted at $245^{-247^{\circ}}$, and left no ash on ignition. The amount of this substance was much too small for further chemical examination, but it was found to possess no marked physiological action, and evidently did not represent the toxic principle of the fruit.

The syrup from which the ethyl acetate liquid was first decanted represented the greater part of the substance extracted by this solvent. It readily reduced Fehling's solution, yielded a crystalline osazone melting at $2 \mathrm{II}-212^{\circ}$, and evidently consisted largely of $i$-glucose.

It was subsequently ascertained, by physiological tests, that the syrup which separated from the ethyl acetate extract was devoid of toxic properties. A small portion of the sawdust, containing the substance which had been subjected to prolonged extraction with ethyl acetate, was then extracted with water, and, as this extract still possessed a high degree of toxicity, it was manifest that the ethyl acetate had removed practically none of the poisonous principle.

With consideration of these results, the dried mixture with sawdust was afterwards extracted for one day in a Soxhlet apparatus with absolute alcohol. The liquid in the flask deposited a quantity of syrup, from which the alcohol was decanted, and as from the latter, on further concentration, an additional quantity of syrup separated, this was also removed from the alcoholic liquid. These preparations, together with an aqueous extract of the sawdust remaining in the extraction apparatus, were all physiologically tested. They were all highly toxic, did not differ appreciably in their action, and nothing crystalline could be obtained from them.

The preliminary examination of Chailletia fruit having shown 
the absence of an alkaloid, and, as so many non-alkaloidal, poisonous substances are glucosides, it was thought probable that the active constituent of the aqueous extract was also a substance of this nature. With the hope of obtaining some confirmation of this view, it was sought to ascertain whether it would yield any definite hydrolytic product. The material contained in the Soxhlet apparatus was, therefore, extracted with water, and in this liquid were dissolved the syrups obtained by the previous extraction with absolute alcohol. The whole was first shaken out several times with ether, which, however, removed only a trace of a brownish, syrupy substance. Sufficient hydrochloric acid was then added to make an approximately 5 per cent. solution, and the mixture boiled for three hours in a flask with an inverted condenser. During the boiling a large quantity of a black amorphous resin separated, and the entire mixture was subsequently distilled in steam. The first drops that passed over were slightly turbid, and had a somewhat empyreumatic odor, but no aldehyde or ketone could be detected in the distillate. The residue in the distilling flask was allowed to cool, and repeatedly shaken with ether. This did not dissolve the black resin, but removed a small quantity of a brownish syrup, which had an unpleasant odor, and, on the addition of water, separated a sticky resin. All the attempts to isolate the toxic principle, or to obtain some definite derivative of it, were, therefore, unsuccessful.

\section{SUMMARY AND CONCLUSIONS.}

The results of the investigation of the fruit of Chailletia toxicaria, Don, as detailed in the preceding pages, may be briefly summarized as follows:

A preliminary examination showed the fruit to contain no alkaloid, cyanogenetic glucoside, or soluble proteid, to which its highly poisonous properties could be attributed. The possible presence of a cyanogenetic glucoside was particularly considered, in view of the recorded statement that such a substance has been isolated from the leaves of the South African species, Chailletia cymosa, Hook. ${ }^{1}$

On extracting 3000 grams of the fruit with light petroleum (boiling-point $40-60^{\circ}$ ), 53 grams, or 1.83 per cent. of a yellowish

1 Compare "Bulletin of the Imperial Institute," I903, Vol. I, p. 14. 
brown fat were obtained. From this a quantity of the mixed triglyceride, oleodistearin, $\mathrm{C}_{3} \mathrm{H}_{5} \cdot\left(\mathrm{C}_{18} \mathrm{H}_{35} \mathrm{O}_{2}\right)_{2} \cdot \mathrm{C}_{18} \mathrm{H}_{33} \mathrm{O}_{2}$, melting-point $43^{\circ}$, was directly separated, and from the residtual fatty matter, after hydrolysis with an alcoholic solution of potassium hydroxide, the following substances were isolated: (I) A small quantity of a phytosterol, $\mathrm{C}_{26} \mathrm{H}_{44} \mathrm{O}$, or possibly a mixture of substances of this class, melting at $135^{-1} 48^{\circ} ;(2)$ Stearic and oleic acids, the latter being apparently associated with an acid having a higher degree of unsaturation; (3) Very small amounts of formic and butyric acids.

The alcoholic extract of the fruit which had previously been deprived of fatty natter, when concentrated and mixed with water, yielded a quantity ( 76 grams) of resinous substance corresponding to 2.5 per cent. of the weight of the fruit. This consisted of a complex mixture, from which nothing crystalline could be obtained. By successive extraction with chloroform, ethyl acetate, and alcohol it was, however, resolved into products which differed essentially in their physiological action. The chloroform extract, for example, had a narcotic or paralytic effect, while the ethyl acetate extract produced delirium and convulsions, and the alcohol extract, although causing nausea, was not distinctly toxic.

The aqueous liquid, separated from the above-mentioned resinous substance, and deprived of tannic and coloring-matters, when concentrated under diminished pressure, formed a thick syrup, which contained a large amount of glucose. This syrup was extremely poisonous, producing, when administered to a dog in relatively small doses, the characteristic action of the fruit, viz., delirium and epileptiform convulsions, soon followed by death. Although many attempts were made to separate the poisonous principle from the syrup by extracting the latter with various solvents, such as ethyl acetate, absolute alcohol, etc., these were unsuccessful, as all the liquids which were capable of removing the active principle also dissolved considerable amounts of sugar.

The physiological experiments connected with this investigation have led to the observation that, with the lower animals, the effects of the poison contained in the Chailletia fruit are only manifested some time after its ingestion. According to the amount administered, a period of several hours may elapse before 
the occurrence of the characteristic convulsions, but, after the onset of the symptoms, death usually follows within fifteen to thirty minutes. A post-mortem examination of the animal shows a condition of cerebral congestion and thrombosis of the superior longitudinal sinus.

It was considered possible that the narcotic effects produced by the chloroform extract of the resin, and the delirium and convulsions caused by the aqueous extract, as above described, are due to the same substance, and that the difference in the symptoms may depend upon the amount of the poison administered or the rate of its absorption. In order to test this assumption successive sub-toxic doses were given to a small dog during a period of several days. Nothing of a definite nature occurred until the tenth day, when the characteristic delirium commenced, followed shortly by convulsions, and death then ensued within about one hour. The post-mortem examination showed a condition of cerebral congestion, and a small patch, apparently of cortical necrosis, near the right crucial sulcus. These experiments thus permit of the following deductions:

(I) That the fruit of Chailletia toxicaria contains at least two active principles, one of which causes cerebral depression or narcosis, and the other cerebral excitation, leading to epileptiform convulsions.

(2) That the poison which causes convulsions is very slowly excreted, so that a cumulative effect is produced by the administration of a series of individually innocuous doses.

It may finally be stated that all the physiological experiments required in the course of this investigation were conducted in the Wellcome Physiological Research Laboratories by Mr. H. H. Dale, and our best thanks are due to him for the valuable assistance he has given us.

\section{ON THE ACTION OF ACID CHLORIDES ON MIXTURES OF AMINES.}

By F. B. DaINs.

Received July 5, sgo6.

In the course of an investigation on the action of acid chlorides on ureas of the type RNHCSNHR', the question arose, which an effort was made to answer, whether when a mixture of two 